\title{
Author Correction: Narrow bounds for the quantum capacity of thermal attenuators
}

\author{
Matteo Rosati (1) ${ }^{1,2}$, Andrea Mari² \& Vittorio Giovannetti ${ }^{2}$
}

Correction to: Nature Communications; https://doi.org/10.1038/s41467-018-06848-0; published online 18 October 2018

The original version of this Article contained an error in Equation (40). The numerator of the fraction inside the logarithm was missing an overall minus sign, and incorrectly read:

$$
Q\left(\Phi_{\eta, N}\right) \leq Q_{\text {twist }}=\max \left\{0, \log _{2} \frac{N(1-\eta)-\eta}{(1+N)(1-\eta)}\right\}
$$

The correct form of Equation (40) is:

$$
Q\left(\Phi_{\eta, N}\right) \leq Q_{\text {twist }}=\max \left\{0, \log _{2} \frac{\eta-N(1-\eta)}{(1+N)(1-\eta)}\right\}
$$

This error has been corrected in both the PDF and HTML versions of the Article.

Published online: 15 January 2019

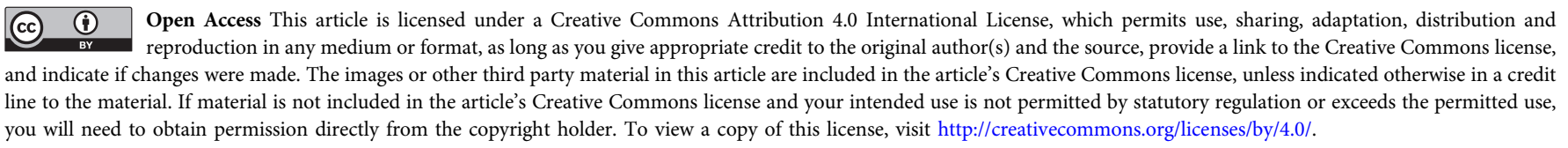

(C) The Author(s) 2019

\footnotetext{
${ }^{1}$ Física Teòrica: Informació i Fenòmens Quàntics, Departament de Física, Universitat Autònoma de Barcelona, 08193 Bellaterra, Spain. ${ }^{2}$ NEST, Scuola Normale Superiore and Istituto Nanoscienze-CNR, 56127 Pisa, Italy. Correspondence and requests for materials should be addressed to M.R. (email: matteo.rosati@uab.cat)
} 